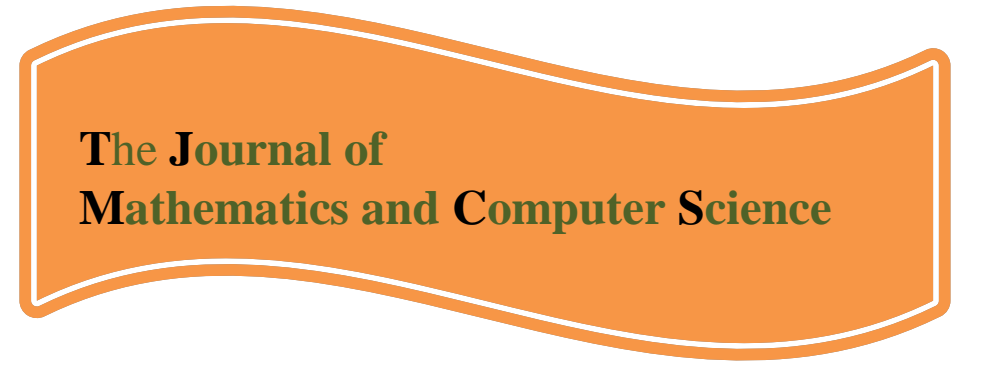

Available online at

\title{
http://www.TIMCS.com
}

\section{Bees Algorithm based Intelligent Backstepping Controller Tuning for Gyro System}

\author{
Reza Gholipour \\ Faculty of Electrical and Computer Engineering, Babol University of Technology, P.0. Box 47135-484, Babol, \\ Iran \\ reza_gh_08@yahoo.com
}

\begin{abstract}
Alireza Khosravi
Faculty of Electrical and Computer Engineering, Babol University of Technology, P.0. Box 47135-484, Babol, Iran akhosravi@nit.ac.ir

\section{Hamed Mojallali}

Electrical Engineering Department, Faculty of Engineering, University of Guilan, P.O. Box 3756, Rasht, Iran mojallali@guilan.ac.ir
\end{abstract}

Received: February 2012, Revised: November 2012

Online Publication: December 2012

\begin{abstract}
In this paper, an intelligent nonlinear controller is presented by intelligent tuning of the backstepping method parameters using Bees Algorithm. The proposed controller is utilized to control of chaos of Gyro system. The backstepping method consists of parameters which could have positive values. The parameters are usually chosen optional by trial and error method. The improper selection of the parameters leads to inappropriate responses or even may lead to instability of the system. The proposed optimal backstepping controller without trial and error determines the parameters of backstepping controller automatically and intelligently by minimizing the Integral of Time multiplied Absolute Error (ITAE) and squared controller output. Finally, the efficiency of the proposed intelligent backstepping controller is illustrated by implementing the method on the Gyro chaotic system.
\end{abstract}

Keywords: Control of chaos; Gyro system; Backstepping method; Bees Algorithm

\section{Mathematics Subject Classification: Primary 54A40; Secondary 46S40.}

\section{Introduction.}

Chaos theory, as a new branch of physics and mathematics, has provided us a new way of viewing the universe and is an important tool to understand the world we live in. Chaotic behaviors have 
been observed in different areas of science and engineering such as mechanics, electronics, physics, medicine, ecology, biology, economy and so on. To avoid troubles arising from unusual behaviors of a chaotic system, chaos control has gained increasing attention in recent years. An important objective of a chaos controller is to suppress the chaotic oscillations completely or reduce them to the regular oscillations [1].

The backstepping approach is one of the most popular nonlinear techniques of control design. It is capable of generating a globally asymptotically stabilizing control laws to suppress and synchronize chaotic system [2]-[5]. The idea of backstepping design is to select recursively some appropriate functions of state variables as pseudo-control inputs for lower dimension subsystems of the overall system. Each backstepping stage results in a new pseudo-control design, expressed in terms of the pseudo-control designs from preceding design stages. When the procedure is terminated, a feedback design for the true control input results, which achieves the original design objective by virtue of a final Lyapunov function, which is formed by summing up the Lyapunov functions associated with each individual design stage.

The Bees Algorithm is a swarm-based optimization procedure that mimics the food foraging behavior of honey bees. The algorithm has been successfully applied to different optimization problems such as continuous function optimization, artificial neural network training, engineering design, multi-objective optimization, data clustering and manufacturing cell formation [6]. Bees Algorithm is used in this paper in order to determine the intelligent backstepping controller parameters. In the proposed controller, the backstepping method parameters are chosen such that the time response of system states converges to zero in a short time, i.e. the system chaos is controlled faster. Besides, more limited control signal is needed for stabilization of system states and chaos control.

The rest of the paper is organized as follows. Section 2 describes the backstepping method. Bees Algorithm is described in Section 3. The proposed intelligent backstepping controller is described in Section 4. In Section 5, simulation results are provided to validate the effectiveness of the proposed method. The paper ends with the conclusion as Section 6 followed by the references.

\section{Backstepping Method}

Considering the following n-order system with strict- feedback form:

$\dot{x}_{i}=f_{i}\left(x_{1}, x_{2}, \ldots, x_{i}\right)+g_{i}\left(x_{1}, x_{2}, \ldots, x_{i}\right) x_{i+1}, 1 \leq \mathrm{i} \leq \mathrm{n}-1$

$\dot{x}_{n}=f_{n}\left(x_{1}, x_{2}, x_{3}, \ldots, x_{n}\right)+g_{n}\left(x_{1}, x_{2}, x_{3}, \ldots, x_{n}\right) u$

Where $x \in R^{n}, u \in R$. With $f_{i}(0)=0$ and $g_{i}(0) \neq 0$ for $i=1, \ldots, n \cdot f_{i}$ and $g_{i}$ are smooth functions and are differentiable.

Step1: Considering the first subsystem of (1), $x_{2}$ is taken as a virtual control input and choose:

$x_{2}=\frac{1}{g_{1}\left(x_{1}\right)}\left(u_{1}-f_{1}\left(x_{1}\right)\right)$

The first subsystem is changed to be $\dot{x}_{1}=u_{1}$. Choosing $u_{1}=-k_{1} x_{1}$ with $k_{1}>0$, the origin of the first subsystem $x_{1}=0$ is asymptotically stable, and the corresponding Lyapunov function is $V_{1}\left(x_{1}\right)=x_{1}^{2} / 2,(2)$ is changed to:

$x_{2}=\phi_{1}\left(x_{1}\right)=\frac{1}{g_{1}\left(x_{1}\right)}\left(-k_{1} x_{1}-f_{1}\left(x_{1}\right)\right)$

Step2: Take $x_{3}$ as a virtual control input and the $\left(x_{1}, x_{2}\right)$ subsystem is changed to (5).

$$
\begin{aligned}
& x_{3}=\frac{1}{g_{2}\left(x_{1}, x_{2}\right)}\left(u_{2}-f_{2}\left(x_{1}, x_{2}\right)\right) \\
& \dot{x}_{1}=f_{1}\left(x_{1}\right)+g_{1}\left(x_{1}\right) x_{2} \\
& \dot{x}_{2}=u_{2}
\end{aligned}
$$

Which is in the form of backstepping method, so the control law $u_{2}$ is as follow: 
$u_{2}=-\frac{\partial V_{1}}{\partial x_{1}} g_{1}\left(x_{1}\right)-k_{2}\left(x_{2}-\phi_{1}\left(x_{1}\right)\right)+\frac{\partial \phi_{1}}{\partial x_{1}}\left[f_{1}\left(x_{1}\right)+g_{1}\left(x_{1}\right) x_{2}\right]$

Where $k_{2}>0$. This control law asymptotically stabilizes $\left(x_{1}, x_{2}\right)=(0,0)$ and Lyapunov function is as (7).

$V_{2}\left(x_{1}, x_{2}\right)=V_{1}\left(x_{1}\right)+\frac{1}{2}\left(x_{2}-\phi_{1}\left(x_{1}\right)\right)^{2}$

Substituting (6) into (4) gives

$x_{3}=\phi_{2}\left(x_{1}, x_{2}\right)=\frac{1}{g_{2}\left(x_{1}, x_{2}\right)}\left[-\frac{\partial V_{1}}{\partial x_{1}} g_{1}\left(x_{1}\right)-k_{2}\left(x_{2}-\phi_{1}\left(x_{1}\right)\right)+\frac{\partial \phi_{1}}{\partial x_{1}}\left(f_{1}\left(x_{1}\right)+g_{1}\left(x_{1}\right) x_{2}\right)-f_{2}\left(x_{1}, x_{2}\right)\right]$

Step n:

Actual control law $u$ where can asymptotically stabilize (1), is as follows:

$$
\begin{aligned}
& u=\frac{1}{g_{n}\left(x_{1}, \ldots, x_{n}\right)}\left[-\frac{\partial V_{n-1}}{\partial x_{n-1}} g_{n-1}\left(x_{1}, \ldots, x_{n-1}\right)-k_{n}\left(x_{n}-\phi_{n-1}\left(x_{1}, \ldots, x_{n-1}\right)\right)+\frac{\partial \phi_{n-1}}{\partial x_{1}}\left(f_{1}\left(x_{1}\right)+\right.\right. \\
& \left.\left.g_{1}\left(x_{1}\right) x_{2}\right)+\ldots+\frac{\partial \phi_{n-1}}{\partial x_{n-1}}\left(f_{n-1}\left(x_{1}, \ldots, x_{n-1}\right)+g_{n-1}\left(x_{1}, \ldots, x_{n-1}\right) x_{n}\right)-f_{n}\left(x_{1}, \ldots, x_{n}\right)\right]
\end{aligned}
$$

Where $k_{n}>0$. This control law asymptotically stabilizes $\left(x_{1}, \ldots, x_{n}\right)=(0, \ldots, 0)$ and Lyapunov function is as (10).

$V_{n}\left(x_{1}, \ldots, x_{n}\right)=V_{n-1}\left(x_{1}, \ldots, x_{n-1}\right)+\frac{1}{2}\left(x_{n}-\phi_{n-1}\left(x_{1}, \ldots, x_{n-1}\right)\right)^{2}$

\section{Bees Algorithm}

The Bees Algorithm is an optimization algorithm inspired by the natural foraging behavior of honey bees to find the optimal solution. Fig. 1 shows the pseudo code for the algorithm in its simplest form. The algorithm requires a number of parameters to be set, namely: number of scout bees $(n)$, number of sites selected out of $n$ visited sites $(m)$, number of best sites out of $m$ selected sites $(e)$, number of bees recruited for best e sites (nep), number of bees recruited for the other ( $m$ $e$ ) selected sites (nsp), initial size of patches (ngh) which includes site and its neighborhood and stopping criterion. The algorithm starts with the $\mathrm{n}$ scout bees being placed randomly in the search space. The fitnesses of the sites visited by the scout bees are evaluated in step 2 .

1. Initialize population with random solutions.

2. Evaluate fitness of the population.

3. While (stopping criterion not met) //Forming new population.

4. Select sites for neighborhood search.

5. Recruit bees for selected sites (more bees for best e sites) and evaluate fitnesses.

6. Select the fittest bee from each patch.

7. Assign remaining bees to search randomly and evaluate their fitnesses.

8. End While.

Figure1. Pseudo code of the basic Bees Algorithm

In step 4 , bees that have the highest fitnesses are chosen as "selected bees" and sites visited by them are chosen for neighborhood search. Then, in steps 5 and 6, the algorithm conducts searches in the neighborhood of the selected sites, assigning more bees to search near to the best e sites. The bees can be chosen directly according to the fitnesses associated with the sites they are visiting. Alternatively, the fitness values are used to determine the probability of the bees being selected. Searches in the neighborhood of the best e sites which represent more promising solutions are made more detailed by recruiting more bees to follow them than the other selected bees. Together with scouting, this differential recruitment is a key operation of the Bees Algorithm.

However, in step 6, for each patch only the bee with the highest fitness will be selected to form the next bee population. In nature, there is no such a restriction. This restriction is introduced here to reduce the number of points to be explored. In step 7 , the remaining bees in the population are 
assigned randomly around the search space scouting for new potential solutions. These steps are repeated until a stopping criterion is met. At the end of each iteration, the colony will have two parts to its new population representatives from each selected patch and other scout bees assigned to conduct random searches [7].

\section{Proposed optimal backstepping controller}

An optimal backstepping controller is formed using Bees Algorithm for the optimization of the backstepping controller key parameters. The proposed controller structure is shown in Fig. 2. In this structure, $x_{i}(i=1,2, \ldots, n)$ are the state variables of the strict-feedback system, $k_{i}(i=1,2, \ldots, n)$ are the backstepping controller parameters and $\mathrm{u}$ is the control signal produced by the optimal backstepping controller. The Bees Algorithm obtains the proper and optimal values of the parameters by minimizing the fitness function.

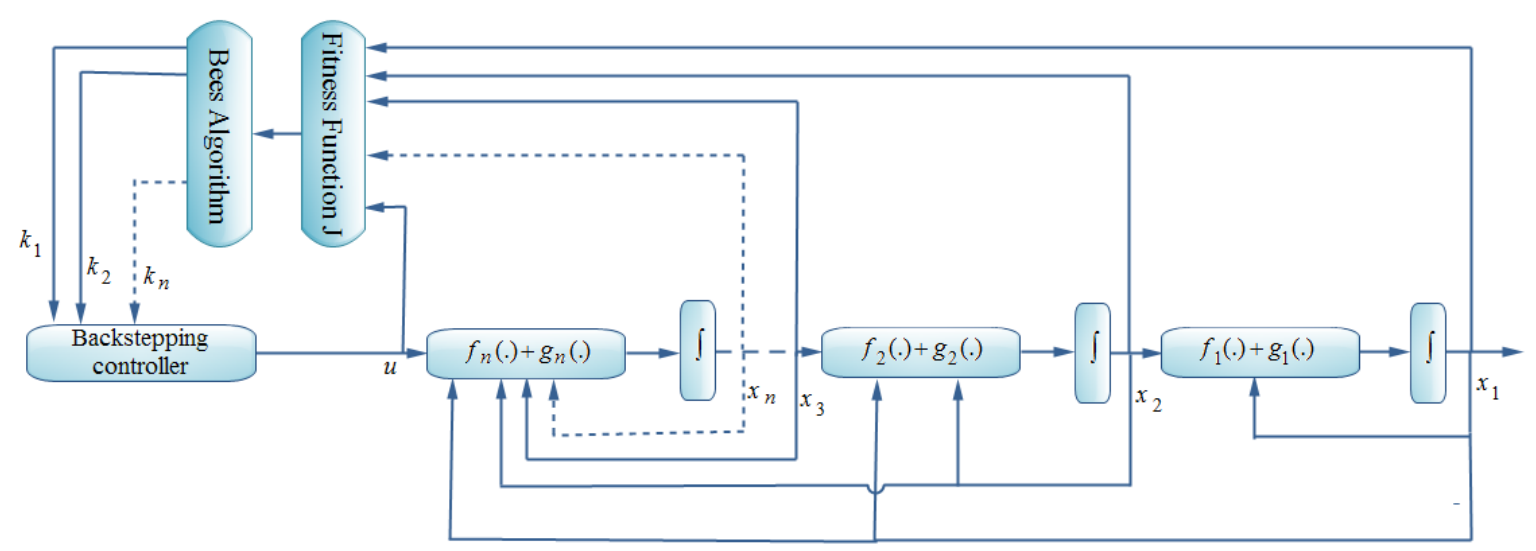

Figure 2. The structure of the proposed controller

Here, the utilized objective function is similar to that of the reference [8]. The objective function is formulized as below:

$J=\int_{0}^{t_{f}}\left[w_{1} t|E(t)|+w_{2} u^{2}(t)\right] d t$; where: $|E(t)|=\sum_{i=1}^{n}\left|e_{i}(t)\right|$ and $e_{i}(t)=x_{i}(t)-x_{d i}$

where $t_{\mathrm{f}}$ is the final time with respect to second, $\mathrm{u}$ is the control signal, $\mathrm{n}$ is the system degree, $\mathrm{x}_{\mathrm{i}}$ $(\mathrm{i}=1,2, \ldots, \mathrm{n})$ are the system state variables, $\mathrm{x}_{\mathrm{di}}(\mathrm{i}=1,2, \ldots, \mathrm{n})$ are the desired trajectories of $\mathrm{x}_{\mathrm{i}}$ $(\mathrm{i}=1,2, \ldots, \mathrm{n})$. According to the goal of stabilizing and chaos control of the system, $\mathrm{x}_{\mathrm{di}}(\mathrm{i}=1,2, \ldots, \mathrm{n})$ is considered to be equal to zero. The weights $w_{1}$ and $w_{2}$ are considered to be equal in this design procedure, in order to assume equal values for two mentioned objectives, i.e. the minimization of the system error is as important as the limitation of the control effort.

\section{Simulation Results}

\subsection{Gyro Chaotic System}

The equation governing the motion of the gyro after necessary transformation is given by [9].

$\left\{\begin{array}{l}\dot{x}_{1}=x_{2} \\ \dot{x}_{2}=g\left(x_{1}\right)-c_{1} x_{2}-c_{2} x_{2}^{3}+(\beta+f \sin \omega t) \sin \left(x_{1}\right) ; \quad g\left(x_{1}\right)=-\alpha^{2} \frac{\left(1-\cos x_{1}\right)^{2}}{\sin ^{3} x_{1}}\end{array}\right.$

Where $\alpha^{2}=100, \quad \beta=1, c_{1}=0.5, \quad c_{2}=0.05$ and $\omega=2$ and $f=35.5$. For the initial condition $\left(x_{1}, x_{2}\right)=(1,-1)$, the chaotic motion of the system is illustrated in Fig. 3 .

\subsection{Controlling Gyro chaotic system}

As shown in Fig. 3, the system has a chaotic behavior, when no control signal is applied. In this section, the backstepping method is utilized for the control of chaos of the Gyro system. For this purpose, a control signal $u$ is added to the equation (12). The system (12) is rewritten, as following: 
R. Gholipour, A. Khosravi, H. Mojallali / TJMCS Vol .5 No.3 (2012) 205 - 211

$\left\{\begin{array}{l}\dot{x}_{1}=x_{2} \\ \dot{x}_{2}=g\left(x_{1}\right)-c_{1} x_{2}-c_{2} x_{2}^{3}+(\beta+f \sin \omega t) \sin \left(x_{1}\right)+u\end{array}\right.$

Backstepping method is used to set states $x_{1}, x_{2}$ to the origin point $(0,0)$ via the control signal $u$ calculated with two steps. According to section (1), the design procedure is as follows:

Step1: $x_{2}$ is taken as (16) to construct the joint Lyapunov function (15) for (14).

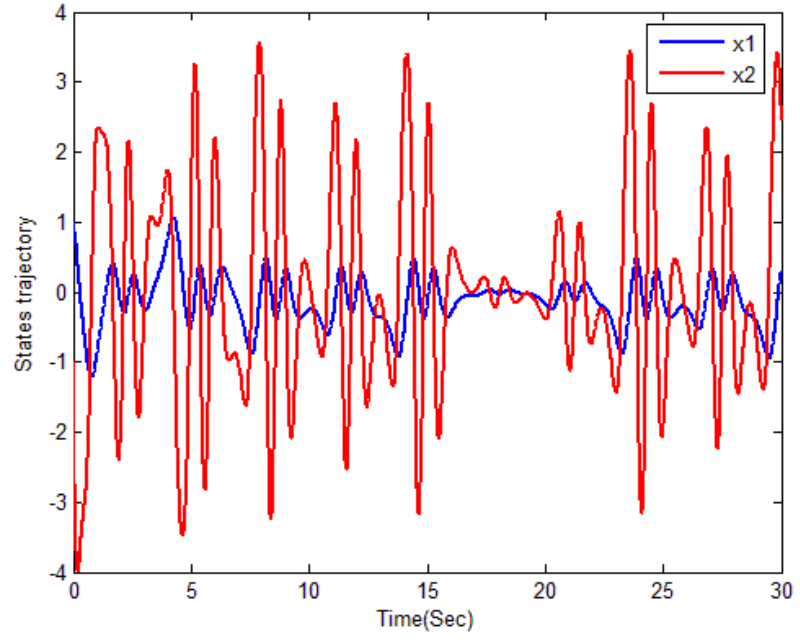

(a)

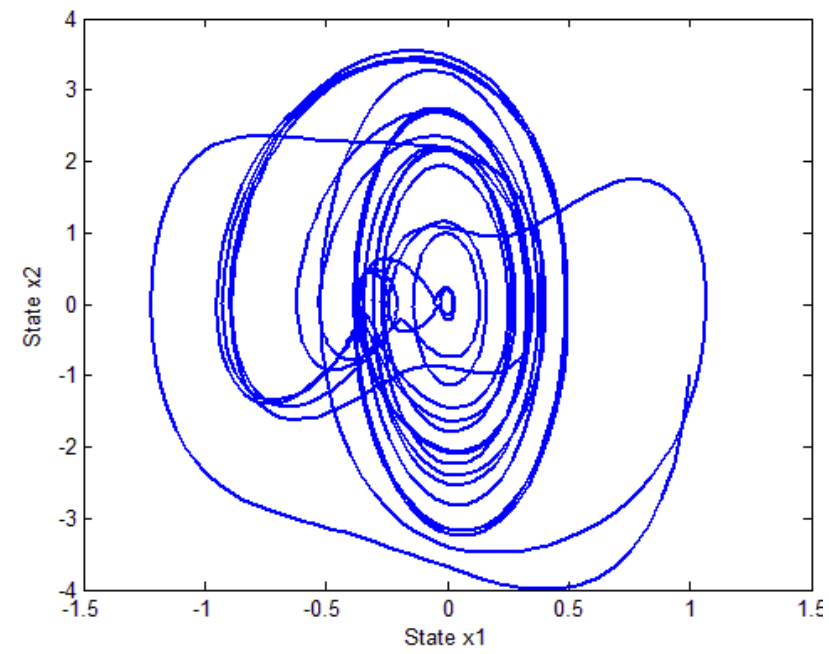

(b)

Figure 3. (a) Chaotic motion (0-30sec) of states, (b) Chaotic attractor after 30sec

$\dot{x}_{1}=x_{2}$

$V_{1}\left(x_{1}\right)=x_{1}^{2} / 2$

$x_{2}=\phi_{1}\left(x_{1}\right)=-k_{1} x_{1}$

Step 2: Final control input and Lyapunov function are given in (18) and (19) for (17).

$\dot{x}_{1}=x_{2}$

$\dot{x}_{2}=g\left(x_{1}\right)-c_{1} x_{2}-c_{2} x_{2}^{3}+(\beta+f \sin \omega t) \sin \left(x_{1}\right)+u$

$u=-\left(1+k_{1} k_{2}\right) x_{1}-\left(k_{1}+k_{2}\right) x_{2}-g\left(x_{1}\right)+c_{1} x_{2}+c_{2} x_{2}^{3}-(\beta+f \sin \omega t) \sin \left(x_{1}\right)$

$V_{2}\left(x_{1}, x_{2}\right)=\frac{1}{2} x_{1}^{2}+\frac{1}{2}\left(x_{2}+k_{1} x_{1}\right)^{2}$

According to equation (18), it is observed that the control signal consists of the parameters which are positive. The Bees Algorithm obtains the proper values of the parameters via minimizing the fitness function. The parameters of the Bees Algorithm are set to the following: $n=20 ; m=10 ; e=5$; $n e p=10 ; n s p=7$ and $n g h=0.01$. The sampling time in this simulation is 0.02 . In proposed controller, the searching ranges for the backstepping parameters $k_{1}$ and $k_{2}$ are limited to [0, 10]. The backstepping parameters are obtained for 20 iterations. In this example $t_{f}$ is equal to 10 seconds. Besides, the weights $w_{1}$ and $w_{2}$ of fitness function are chosen as 0.5. $n$ represents the system degree and is equal to 2 in this example.

The parameters of backstepping controller are obtained by using Bees Algorithm, as follows: $k_{1}=7.1313, k_{2}=7.1209$. The search process of Bees Algorithm for finding the parameters is shown in Fig. 4(a). Besides, the fitness value obtained by the algorithm is 20.8420 . The trajectory of fitness variations with respect to algorithm iteration is shown in Fig. 4(b). 
The time response of the states of Gyro system after applying the controller is shown in Fig 5(a). The controlled chaos of the system is demonstrated in Fig. 5(b). Also, the control signal is illustrated in Fig. 5(c). As shown in Figs. 5(a) and 5(b), the Bees Algorithm causes the time response of the states of the system converge to zero in a shorter time by minimizing the fitness function. In addition, according to Fig. 5(c), it is observed that the proposed controller has created a limited control signal to chaos control of Gyro system.

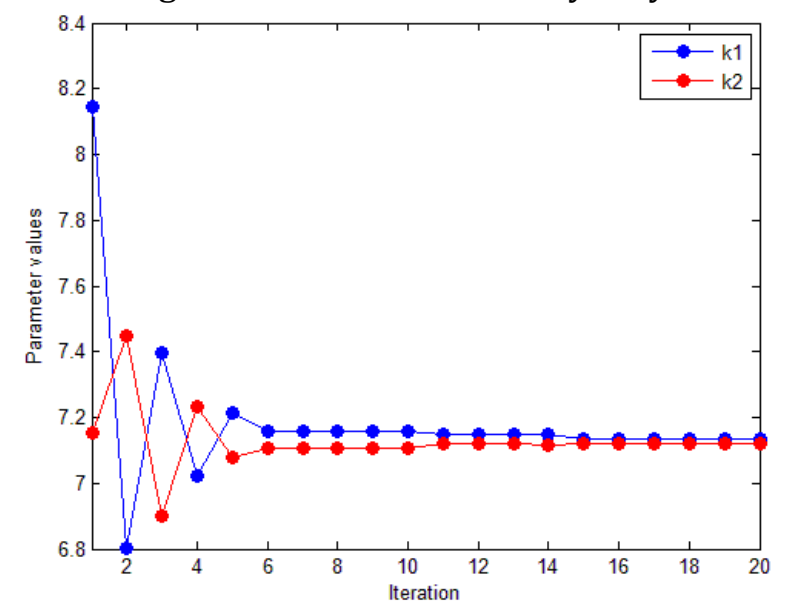

(a)

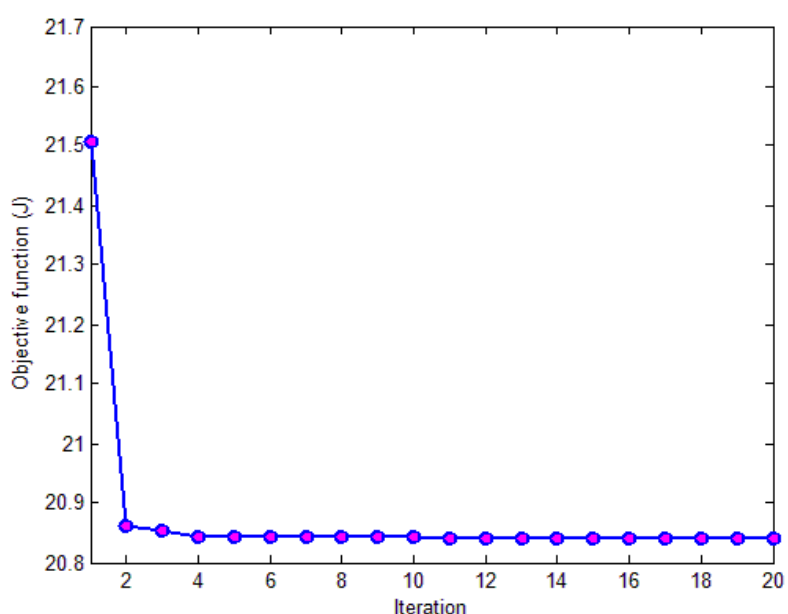

(b)

Figure 4. (a) The parameter values trajectory, (b) The Objective function trajectory

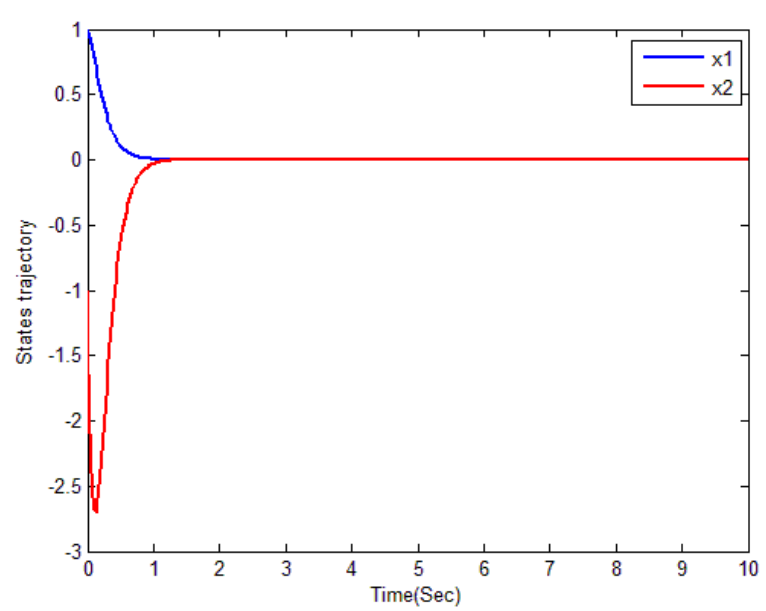

(a)

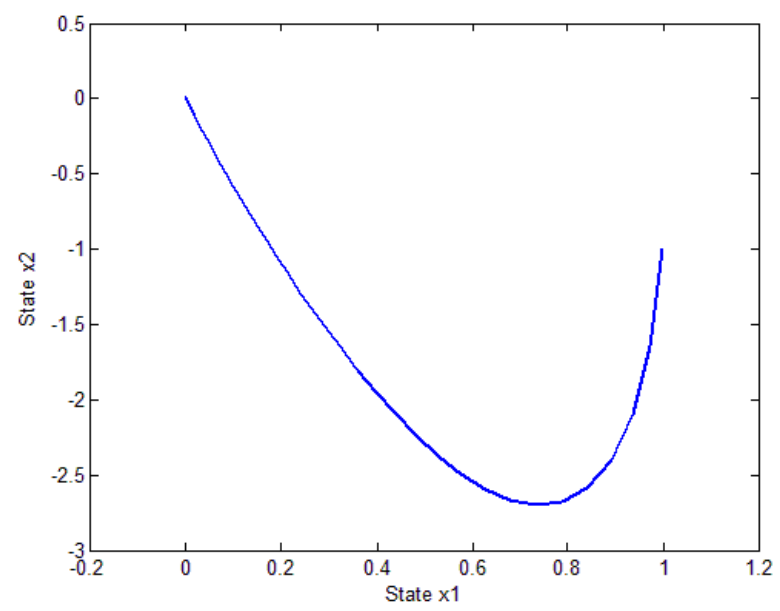

(b)

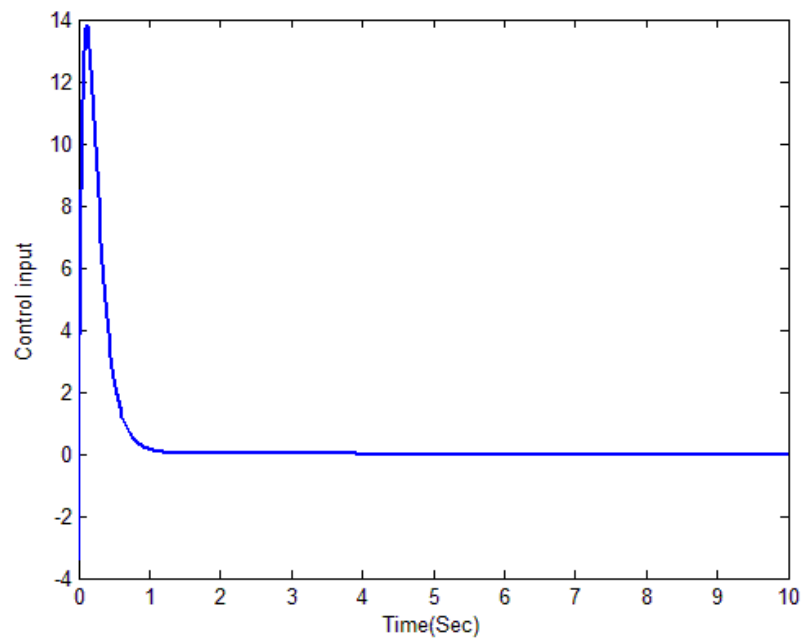

(c)

Figure 5. (a) Controlled time response of the states, (b) Controlled Chaotic attractor (0-30sec), (c) The control law 


\section{Conclusion}

This paper has proposed an optimal backstepping controller for chaos control of the Gyro chaotic system. A weighted sum of the Integral of Time multiplied Absolute Error and squared control signal is the minimized fitness function via the Bees Algorithm. Fast control of chaos in a very short time and having more limited control signal for this purpose, are the great advantages of the proposed controller. Numerical simulations are presented to show the effectiveness of the proposed scheme.

\section{References.}

[1] M.S. Tavazoei and M. Haeri, Chaos control via a simple fractional-order controller, Physics Letters A, 372 (2008), 798-807.

[2] J. Lu and S. Zhang, Controlling Chen's chaotic attractor using backstepping design based on parameters identification, Phys Lett A, 286 (2001), 145-149.

[3] J.H. Park, Synchronization of Genesio chaotic system via backstepping approach, Chaos, Solitons \& Fractals, 27 (2006), 1369-1375.

[4] S. Bowong and F.M. Moukam Kakmeni, Chaos control of uncertain chaotic systems via backstepping approach, ASME J Vibrat Acoust, 128 (2006), 21-27.

[5] M.T. Yassen, Chaos control of chaotic dynamical systems using backstepping design", Chaos, Solitons \& Fractals, 27 (2006), 537-548.

[6] D.T. Pham and M. Kalyoncu, Optimization of a Fuzzy Logic Controller for a Flexible Single-Link Robot Arm Using the Bees Algorithm, 2009 7th IEEE International Conference on Industrial Informatics (INDIN 2009), 475-480.

[7] D.T. Pham, A. Ghanbarzadeh, E. Koç, S. Otri, S. Rahim and M. Zaidi, The Bees Algorithm - A Novel Tool for Complex Optimisation Problems, Proc. of the 2nd Virtual Int. Conf. on Intelligent Production Machines and Systems (IPROMS 2006), Elsevier (Oxford), 454-459.

[8] I. Pan, S. Das and A. Gupta, Tuning of an optimal fuzzy PID controller with stochastic algorithms for networked control systems with random time delay, ISA Transactions, 50 (2011), 28-36.

[9] H.K. Chen, Chaos and chaos synchronization of a symmetric gyro with linear-plus-cubic damping, Journal of Sound and Vibration, 255 (2002), 719-740. 